\title{
Preliminary Investigations into the Evolution of Cooperative Strategies in a Minimally Spatial Model
}

\author{
Simon T. Powers and Richard A. Watson \\ School of Electronics and Computer Science, University of Southampton \\ Southampton, SO17 1BJ, U.K. \\ stp05r@ecs.soton.ac.uk, raw@ecs.soton.ac.uk
}

\begin{abstract}
In this paper we outline a simple model of spatially structured populations that is an extension of the replicator dynamics approach used in evolutionary game theory. Using this model, we are able to investigate issues such as how the degree of spatial localisation affects the evolution of cooperative and selfish genotypes in a resource sharing scenario.
\end{abstract}

Categories and Subject Descriptors: I.6.5 [Simulation and Modeling]: Model Development-Modeling methodologies

General Terms: Algorithms

Keywords: Evolutionary dynamics, evolutionary game theory, spatial model, resource sharing, cooperation

\section{INTRODUCTION}

Frequency-dependant fitness models based on evolutionary game theory typically assume that a member of a population interacts with every other member of that population with equal frequency [2]. However, this assumption is invalid in many cases and represents a worst-case scenario for the evolution of cooperative traits. On the other hand, a large body of literature has shown how spatial structure, by limiting with whom individuals interact, can promote cooperation. In these works, spatial structure is typically modelled using an individual-based simulation technique in which the individuals occupy a grid-world (e.g. $[3,1])$. We argue that such models are more complex than they need to be, as they require a large number of assumptions and parameter settings that can introduce unintended artefacts into the results.

In this research programme, we aim to combine the simplicity of replicator dynamics equations with the ability of individual-based approaches to model spatial structure. Our approach is based on grouping individuals with the same genotype into an homogeneous colony that grows by consumption of a limiting resource. We then consider two resource consumption strategy genotypes: a selfish genotype that replicates at a faster rate but that uses resource inefficiently, and a cooperative genotype that replicates at a slower rate while consuming resource more efficiently. Thus, although the cooperative strategy can sustain more individuals under limited resource, it is at a disadvantage when resource is freely shared.

Copyright is held by the author/owner(s). GECCO'07, July 7-11, 2007, London, England, United Kingdom. ACM 978-1-59593-697-4/07/0007.

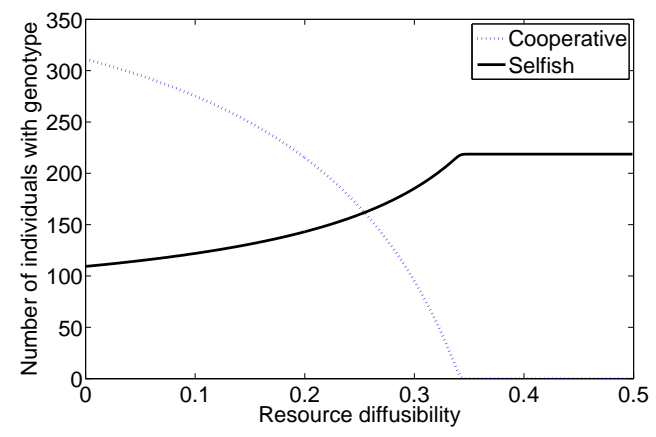

Figure 1: Number of individuals carrying each genotype at equilibrium.

Spatial localisation of interactions is introduced by providing each colony with its own resource supply that is partially segregated from the other colony. The degree of resource segregation is analogous to the degree of spatial localisation, i.e. whether an individual interacts with all members of the population or only with its neighbours. Specifically, our model contains a single parameter, resource diffusibility, that determines how segregated each colony's resource supply is. By introducing spatial localisation as degree of movement of resource between homogeneous colonies, it is still possible to state our model entirely as a series of coupled difference equations. This makes it significantly simpler and easier to analyse than an individual-based approach.

\section{RESULTS}

Results showing the effect of varying the degree of resource diffusibility on genotype coexistence are illustrated in Figure 1. They show that coexistence of both genotypes is possible for a range of degrees of resource diffusibility, and that for most of this range the cooperative genotype has the greater frequency. These results also agree with those found in more complex models $[3,1]$. In future work, we intend to investigate invadability dynamics.

\section{REFERENCES}

[1] J.-U. Kreft. Biofilms promote altruism. Microbiology, 150:2751-2760, 2004.

[2] J. Maynard Smith. Evolution and the Theory of Games. Cambridge University Press, 1982.

[3] T. Pfeiffer, S. Schuster, and S. Bonhoeffer. Cooperation and competition in the evolution of ATP-producing pathways. Science, 292(5516):504-507, 2001. 